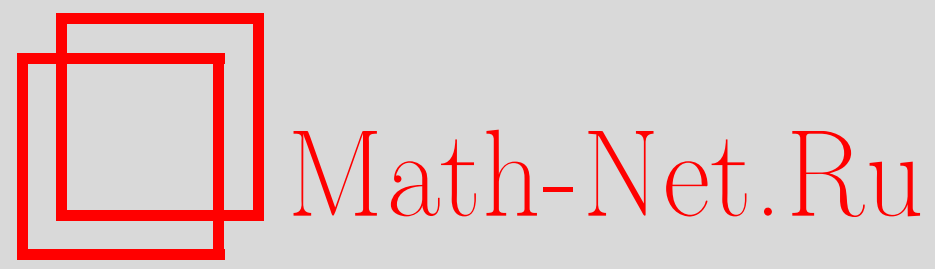

А. А. Карацуба, Эйлер и теория чисел, Совр. пробл. матем., 2008, выпуск 11, 19-37

DOI: https://doi.org/10.4213/spm21

Использование Общероссийского математического портала MathNet.Ru подразумевает, что вы прочитали и согласны с пользовательским соглашением

http://www . mathnet.ru/rus/agreement

Параметры загрузки:

IP : 54.198 .64 .247

26 апреля 2023 г., 18:36:31

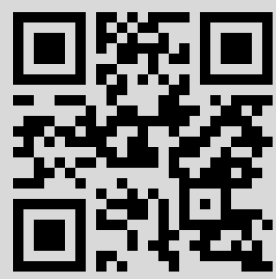




\title{
Эйлер и теория чисел
}

\author{
А. А. Карацуба
}

Здесь излагается расширенное содержание доклада, прочитанного автором на конференции, посвященной 300-летию со дня рождения Л. Эйлера (Математический институт им. В. А. Стеклова, 17 мая 2007 г.)

А) Седьмая часть научных работ Эйлера посвящена теории чисел (из 900 названий статей Эйлера и его сына Иоганна Альбрехта 120 посвящены теории чисел).

Б) Внимание Эйлера к теории чисел привлек Гольдбах (письмо Гольдбаха к Эйлеру от 1 декабря 1729 г.), и первой работой была работа о теореме Ферма, касающаяся представимости простых чисел суммою двух квадратов целых чисел. Несмотря на разницу в возрасте в 17 лет, Гольдбах и Эйлер дружили, вели активную переписку, где ставились и решались проблемы теории чисел, вплоть до кончины Гольдбаха в 1764 г.

В) Самым значительным изданным трудом Эйлера по теории чисел являются его "Арифметические сочинения". Это двухтомная монография, изданная в 1849 г. в Петербурге под редакцией Фуса, Буняковского, Чебышева. К изданию трудов Эйлера привлек Чебышева в 1847 г. Буняковский. После этого издания Чебышев занялся теорией чисел и написал две свои знаменитые статьи по теории чисел.

Г) Уже в наше время (1997 г.) вышла книга (см. [4]) "Неопубликованные материалы Л. Эйлера по теории чисел" под редакцией Н. И. Невской, где содержатся заметки из записных книжек Эйлера, касающиеся вопросов теории чисел.

Д) Во времена Эйлера еще не было науки "теория чисел". Да и сама математика, как строгая наука, начинала только-только строиться. Как самостоятельная наука теория чисел сформировалась значительно позднее, после работ Гаусса, после издания его классического труда “Арифметические исследования" (1801г.).

Е) Но в то же время период деятельности Эйлера был тем периодом, когда закладывался фундамент теории чисел, когда находились (создавались) понятия, ставились и решались задачи, которые впоследствии составили здание теории чисел.

(c) Карацуба А. А., 2008 
Я расскажу о некоторых, на мой взгляд, самых важных, результатах, полученных Эйлером в теории чисел.

1. Со времен Пифагора (VI в. до н. э.) стоял вопрос о совершенных и дружественных числах. Натуральное число $а$ называется совершенным, если сумма всех его собственных делителей, т.е. делителей, отличных от $a$, равняется $a$ (таким образом, сумма всех делителей $а$ равняется 2a). Например, число 6 является совершенным, так как собственные делители 6 есть $1,2,3$ и $1+2+3=6$. До Эйлера была теорема Евклида: если $a=\left(2^{p}-1\right) 2^{p-1}$ и число $2^{p}-1$ является простым, то $a-$ совершенное число. Например, при $p=2$ получаем: $2^{p}-1=2^{2}-1=3$, $a=6$; при $p=3$ получаем: $2^{p}-1=2^{3}-1=7, a=28$. Простые числа $M_{p}=2^{p}-1$ называются простыми Мерсенна. Эйлер доказал, что если число $a$ является четным совершенным числом, то оно имеет указанный выше вид. Таким образом, для того, чтобы четное число $а$ было совершенным, необходимо и достаточно, чтобы оно имело следующий вид:

$$
a=M_{p} 2^{p-1}, \quad M_{p}=2^{p}-1-\text { простое число. }
$$

Эйлер высказал гипотезу, что нет нечетных совершенных чисел (гипотеза не доказана, 2007 г.).

Натуральные числа $A$ и $B$ называются дружественными, если сумма собственных делителей $A$ равняется $B$ и наоборот, сумма собственных делителей $B$ равна $A$. До Эйлера были известны две пары дружественных чисел: пара Пифагора $(220,284)$ и пара Ферма-Декарта $(17296,18416)$. Эйлер нашел 59 новых пар, в частности, пары нечетных дружественных чисел; например, такой парой будет $\left(3^{2} \cdot 7 \cdot 13 \cdot 5 \cdot 17,3^{2} \cdot 7 \cdot 13 \cdot 107\right)$.

2. Как было сказано выше, первой работой Эйлера по теории чисел была работа, связанная с теоремой Ферма. Эйлер хорошо был знаком с работами Ферма и под их влиянием написал целый ряд своих.

а) Ферма рассматривал числа $F_{n}=2^{2^{n}}+1$ и высказал гипотезу, что все они, $n=0,1,2, \ldots$ являются простыми. Эйлер опроверг эту гипотезу, доказав, что $F_{5}$ делится на 641 . Сейчас числа $F_{n}$ называются числами Ферма. Еще не найдено ни одного простого $F_{n}, n \geqslant 5$. Харди высказал гипотезу, что только конечное число чисел $F_{n}$ являются простыми (гипотеза не доказана, 2007 г.). 
б) Ферма принадлежит следующая теорема ("малая теорема Ферма"): если $p$ - простое число, $a$ не делится на $p$, то $a^{p-1}-1$ делится на $p$.

Эта теорема играет одну из главных ролей в элементарной теории чисел и алгебре. Эйлер нашел принципиально новое обобщение малой теоремы Ферма. При натуральном числе $n$ Эйлер определил функцию $\varphi(n)$ ("функция Эйлера") - количество натуральных чисел, не превосходящих $n$ и взаимно-простых с $n$. Теорема Эйлера: если $a$ и $n-$ взаимно-простые числа, то $a^{\varphi(n)}-1$ делится на $n$. Функция Эйлера и теорема Эйлера занимают одно из центральных мест в современной теории чисел. Буква $\varphi$ и символ $\varphi(n)$ введены Гауссом.

в) Эйлером доказаны основные теоремы теории сравнений, в частности, теоремы о квадратичных вычетах. Например, им доказано соотношение ("критерий Эйлера"), которое в современной форме записывается так:

$$
\left(\frac{a}{p}\right) \equiv a^{\frac{p-1}{2}}(\bmod p)
$$

Здесь $p$ - нечетное простое число, $(a / p)$ - символ Лежандра,

$$
\left(\frac{a}{p}\right)=\left\{\begin{aligned}
+1, & \text { если } a-\text { квадратичный вычет по модулю } p ; \\
0, & \text { если } a-\text { делится на } p ; \\
-1, & \text { если } a-\text { квадратичный невычет по модулю } p .
\end{aligned}\right.
$$

Эйлер нашел и сформулировал квадратичный закон взаимности: если $p$ и $q$ - нечетные простые числа, то

$$
\left(\frac{p}{q}\right)\left(\frac{q}{p}\right)=(-1)^{\frac{p-1}{2} \cdot \frac{q-1}{2}}
$$

Квадратичный закон взаимности был доказан Гауссом (им дано восемь разных доказательств этого утверждения). Обобщения закона взаимности даны Гауссом, Эйзенштейном, Куммером, Гильбертом, Артином, Хассе, Шафаревичем, Востоковым.

г) Вслед за Ферма, Эйлер много внимания уделил вопросам представимости чисел значениями бинарных квадратичных форм. Ферма предположил, а доказал Эйлер, что простые числа вида $p=4 n+1$ и только такие представимы в виде $x^{2}+y^{2}$ и указанное представление единственное. Эйлер доказал, что простые 
числа вида $p=8 n+1, p=8 n+3$ и только такие, представимы в виде $x^{2}+2 y^{2}$, простые числа вида $p=6 n+1$ и только такие представимы в виде $x^{2}+3 y^{2}$, причем указанные представления единственные.

д) Эйлер исследовал уравнение $y^{2}-D x^{2}=1, D \neq k^{2}$, которое сейчас называется уравнением Пелля, указал алгоритм нахождения его решений через цепные дроби $\sqrt{D}$ и для всех $D<100$ привел наименьшие решения этого уравнения.

е) Продолжением г) явились работы Эйлера по представлению натуральных чисел суммами квадратов. Занимаясь этой проблемой, Эйлер доказал свое знаменитое тождество: произведение суммы четырех квадратов на сумму четырех квадратов равняется сумме четырех квадратов. Он был близок к доказательству теоремы о том, что любое натуральное число есть сумма четырех квадратов целых чисел (теорема Лагранжа). Это ясно видно по его записям в записных книжках. После того, как Лагранж доказал теорему о четырех квадратах, Эйлер вернулся к этой тематике и дал новое, более простое и прямое доказательство теоремы Лагранжа.

Хочу обратить внимание слушателей на одну удивительную проблему, которая идет от Эйлера и связана с суммами квадратов целых чисел.

Все натуральные числа, за исключением чисел вида $4^{m}(8 n+7)$, $m, n=0,1,2 \ldots$, представимы в виде $x^{2}+y^{2}+z^{2}$. Естественно возникает вопрос: как близко к заданному числу $N$ можно подойти суммой двух квадратов целых чисел? Так как $(n+1)^{2}-n^{2}=2 n+1$ и правая часть этого равенства имеет порядок корня квадратного из $n^{2}$, то одним квадратом можно подойти к $N$ на расстояние порядка $\sqrt{N}$. Следовательно, суммой двух квадратов можно подойти к $N$ на расстояние порядка $\sqrt[4]{N}$. А можно ли подойти ближе? Со времен Эйлера стоит эта задача "без движения", хотя есть гипотеза о том, что

$$
\min _{x, y \in \mathbb{Z}}\left|N-x^{2}-y^{2}\right| \leqslant N^{\varepsilon}
$$

где $\varepsilon>0, \varepsilon-$ любое, $N \geqslant N_{1}(\varepsilon)$. Заменить $\frac{1}{4}$ в предыдущем рассуждении на $\frac{1}{4}-c$ со сколь угодно малым фиксированным $c>0$, не могут, и эта, на первый взгляд, простая задача "стоит на месте" более 250 лет.

ж) Эйлер размышлял над Великой теоремой Ферма. Он доказал, что уравнение $x^{3}+y^{3}=z^{3}$ не имеет решений в натуральных 
числах $x, y, z$. Метод его доказательства опирался на арифметику алгебраических чисел. В частности, из тождества

$$
p^{2}+3 q^{2}=(p+q \sqrt{-3})(p-q \sqrt{-3})=r^{3},
$$

он делает вывод, что $p \pm q \sqrt{-3}=(u \pm v \sqrt{-3})^{3}$, т.е. пользуется неявно основной теоремой арифметики (однозначность разложения натуральных чисел на простые сомножители) в кольце целых чисел $u \pm v \sqrt{-3}$. Но в то время не было основной теоремы арифметики (только Гаусс доказал эту теорему) и не возникало соответствующих вопросов. Но идея строить арифметику алгебраических чисел и применять ее к доказательству Великой теоремы Ферма принадлежит Эйлеру. Эта идея продолжила свое развитие в работах Гаусса, Куммера, Дирихле, Дедекинда, Золотарева, Кронекера, Гильберта. Была построена большая наука - алгебраическая теория чисел, которая получила основное свое развитие в 20-м веке.

3. Эйлер ввел в математику первообразные корни и индексы (дискретный аналог логарифма), которые в наше время нашли многочисленные применения в криптографии.

4. Эйлер был одним из первых, кто понял, что иррациональности бывают разной природы - более "простые", такие как $\sqrt{2}$, и более "сложные", такие, как $\log _{2} 3$. В своей монографии "Введение в анализ бесконечно малых" он пишет: "Так как логарифмы чисел, не являющиеся степенями основания $a$, не могут быть выражены ни рационально, ни иррационально, то они справедливо относятся к количествам трансцендентным; поэтому логарифмы обычно причисляются к количествам трансцендентным" (с. 104, п. 105).

В то время еще не было точного определения трансцендентного числа. Оно появилось через 100 лет, в 1844 г. его ввел Лиувилль. Он же привел примеры трансцендентных чисел. В 1873 г. Эрмит доказал трансцендентность числа $e$, а в 1882 г. Линдеман доказал трансцендентность числа $\pi$, чем была решена проблема квадратуры круга. В 1900 г. Гильберт среди своих 23-х проблем сформулировал утверждение Эйлера в несколько измененной форме как 7-ю проблему: если $\alpha$ - алгебраическое число, $\alpha \neq 0,1, \beta$ - алгебраическая иррациональность, то $\alpha^{\beta}$ - трансцендентное число. Первый результат в решении этой проблемы получил Гельфонд в 1929 г., затем - Кузьмин (1930 г.). Полностью 
проблему решили в 1934 г. Гельфонд и, независимо, Шнайдер. Была создана большая наука - теория трансцендентных чисел, с большим количеством глубоких результатов об алгебраических и трансцендентных числах и с многочисленными приложениями к диофантовым уравнениям (Туэ, Зигель, Рот, Бейкер, Спринджук, Нестеренко, Бертран и др.).

5. Эйлер, как и его предшественники (Пифагор, Евклид, Ферма), размышлял над простыми числами. Как уже было сказано, он доказал целый ряд замечательных теорем о представлении простых чисел значениями бинарных квадратичных форм. Он высказал гипотезу о том, что между $a$ и $2 a, a \geqslant 2$, всегда есть простое число. Эта гипотеза стала называться "Постулат Бертрана" и была доказана в 1848 г. Чебышевым.

Еще одна проблема теории простых чисел прямо связана с Эйлером. Легко доказать, что нет многочлена с целыми коэффициентами, все значения которого при целых значениях аргумента являются простыми числами. В то же время, повидимому, со времен Пифагора, стоит проблема о том, что в последовательности чисел $n^{2}+1, n=1,2,3, \ldots$, содержится бесконечно много простых чисел. Эйлер рассмотрел многочлен $f_{1}(x)=x^{2}-x+41$, который при $x=0,1,2, \ldots, 40$ принимает значения простых чисел. В 1939 г. Бигер рассмотрел многочлен $f_{2}(x)=x^{2}-x+72491$, который при $x=0,1,2, \ldots, 11000$ принимает значения 4923 простых чисел. Возникла гипотеза (Хуа Ло-кен, 1940 г.): для каждого $N \geqslant 1$ существует целое число $A$ такое, что многочлен $f(x)=x^{2}-x+A$ при $x=0,1,2, \ldots, N$ принимает значения простых чисел (гипотеза не доказана, 2007 г.).

В переписке с Гольдбахом была поставлена Проблема Гольдбаха (тернарная П.Г. или просто П.Г.) и Проблема Эйлера (бинарная П.Г.): каждое нечетное число $\geqslant 9$ есть сумма трех нечетных простых чисел и каждое четное число $\geqslant 6$ есть сумма двух нечетных простых чисел. П.Г. была решена в 1937 г. Виноградовым.

Уже из сказанного видно, насколько велик вклад Эйлера в теорию чисел.

6. Но самое главное, что внес в теорию чисел Эйлер, это применение анализа для решения задач теории чисел. Эйлеру принадлежат два метода решения задач теории чисел с помощью анализа, которые послужили основой той большой науки, которая сейчас называется аналитической теорией чисел. Основы, первые 
идеи применения анализа к решению задач теории чисел содержатся в монографии Эйлера "Введение в анализ бесконечно малых", 1748 г. (см. [1]).

7. Совершенно необыкновенным является труд Эйлера, названный им "Введение в анализ бесконечно малых" (коротко "Введение"). Иногда сравнивают и проводят параллель между творчеством Баха и Эйлера, подчеркивая широту, глубину, оригинальность, простоту и красоту созданных ими произведений. Если внимательно посмотреть на сочинения Баха и Эйлера, то можно заметить большое сходство "Хорошо темперированного клавира" Баха и "Введения" Эйлера.

Как первое, так и второе сочинения, по первоначальному замыслу авторов, должны были быть учебными пособиями для начинающих. Но созданные шедевры вышли далеко за рамки учебников. В них содержатся поистине удивительные открытия и основы целых новых направлений, соответственно, в музыке и математике.

Сходство видно уже в предисловиях к этим сочинениям. Заглавие цикла на титульном листе у Баха звучит так: "Хорошо темперированный клавир, или Прелюдии и фуги во всех тонах и полутонах, касающихся как терций мажорных, или До, Ре, Ми, так и терций минорных, или Ре, Ми, Фа. Для пользы и употребления жадного до учения музыкального юношества, как и для особого времяпрепровождения тех, кто уже преуспел в этом учении, составлено и изготовлено Иоганном Себастьяном Бахам. В году 1722

У Эйлера в "Предисловии" к "Введению" написано: "Такое разнообразие материала легко могло разрастись на много томов; но я дал все, по мере возможности, настолько сжато, что всюду излагается - весьма впрочем, ясно - лишь основное; более же подробная разработка предоставляется трудолюбию читателей, дабы они имели на чем упражнять свои силы, чтобы еще шире раздвинуть границы Анализа. Не боюсь открыто заявить, что в этой книге не только содержится много совершенно нового, но также указаны источники, откуда можно черпать многие замечательные открытия".

8. И первый, один из самых знаменитых источников, это глава XV "О рядах, возникающих при перемножении сомножителей", c. 238-260. Остановлюсь только на одном результате этой главы. 
Результат относится к введенной Эйлером функции, которая впоследствии стала называться дзета-функцией Римана. На с. 241 Эйлер выписывает соотношения: “. . . если взять какую-либо степень простых чисел, ...

$$
P=\frac{1}{\left(1-\frac{1}{2^{n}}\right)\left(1-\frac{1}{3^{n}}\right)\left(1-\frac{1}{5^{n}}\right)\left(1-\frac{1}{7^{n}}\right)\left(1-\frac{1}{11^{n}}\right) \quad \text { и т.Д. }},
$$

то получим

$$
P=1+\frac{1}{2^{n}}+\frac{1}{3^{n}}+\frac{1}{4^{n}}+\frac{1}{5^{n}}+\frac{1}{6^{n}}+\frac{1}{7^{n}}+\frac{1}{8^{n}}+\text { и т.д., }
$$

где встречаются все без исключения натуральные числа".

Правая часть последнего равенства сейчас обозначается символом $\zeta(n)$. Таким образом, при $s=n$ получаем:

$$
\zeta(s)=\sum_{k=1}^{\infty} \frac{1}{k^{s}}=\prod_{p}\left(1-\frac{1}{p^{s}}\right)^{-1} .
$$

Равенство (1) называется тождеством Эйлера. Оно играет в настоящее время главную роль при изучении законов распределения простых чисел. Сам Эйлер, пользуясь (1), дал новое, аналитическое, т.е. с привлечением средств анализа, доказательство теоремы Евклида о бесконечности количества простых чисел. Действительно, если простых чисел - конечное число, то правая часть (1) при $s \rightarrow 1+0$ стремится к конечному пределу, именно, к числу

$$
\prod_{p}\left(1-\frac{1}{p}\right)^{-1}
$$

Левая часть (1) при $s \rightarrow 1+0$ стремится к гармоническому ряду, т.е. к бесконечности. Получили противоречие.

Это открытие Эйлера послужило созданию того большого направления аналитической теории чисел, которое занимается вопросами, связанными с простыми числами. И здесь, главную роль, как об этом сказано выше, играет тождество Эйлера.

Идею Эйлера доказывать бесконечность множества простых чисел с помощью тождества (1) применил в 1837 г. Дирихле к доказательству теоремы о бесконечности множества простых чисел, принадлежащих арифметической прогрессии с разностью $k$ и первым членом $l$, где $1 \leqslant l<k,(l, k)=1$. Для этого он ввел 
(нашел) функции $\chi(n)$, определенные на множестве целых чисел $n$ и удовлетворяющие следующим свойствам: $\chi(1)=1 ; \chi(n)$ - периодическая с периодом $k$, т.е. $\chi(n+k)=\chi(n) ; \chi(n)$ - мультипликативная функция, т.е. $\chi(n m)=\chi(n) \chi(m)$. Функции $\chi(n)$ стали называться "характерами Дирихле". При заданном числе $k$ ("модуль характера") существует ровно $\varphi(k)$ разных характеров. Среди этих характеров есть один - главный характер $\chi_{0}(n)$, который равен 1 при $(n, k)=1$, и равен 0 при $(n, k)>1$. При помощи характеров можно аналитически, т.е. формульно, выделять числа $n$, принадлежащие заданной арифметической прогрессии, так как выполняется следующее равенство ("ортогональность характеров"):

$$
\frac{1}{\varphi(k)} \sum_{\chi \bmod k)} \chi(n) \chi\left(l_{1}\right)= \begin{cases}1, & \text { если } n l_{1} \equiv 1(\bmod k) ; \\ 0, & \text { если } n l_{1} \neq 1(\bmod k) .\end{cases}
$$

В последней сумме $l_{1} l \equiv 1(\bmod k)$ и суммирование ведется по всем характерам модуля $k$.

При $s>1$ Дирихле рассмотрел аналог функции $\zeta(s)$ - функцию $L(s, \chi)$ ("эль-функция Дирихле"):

$$
L(s, \chi)=\sum_{n=1}^{\infty} \frac{\chi(n)}{n^{s}}=\prod_{p}\left(1-\frac{\chi(p)}{p^{s}}\right)^{-1} .
$$

Нетрудно видеть, что $L\left(s, \chi_{0}\right)$ отличается от $\zeta(s)$ множителем, равным

$$
\prod_{p \mid k}\left(1-\frac{1}{p^{s}}\right)
$$

т.е. $L\left(s, \chi_{0}\right) \rightarrow+\infty$ при $s \rightarrow 1+0$. Кроме того, Дирихле доказал, что $L(1, \chi) \neq 0$, если $\chi \neq \chi_{0}$. Из этого и соотношений $(2)$ и $(3)$ легко следует, что

$$
\sum_{p \equiv(\bmod k)} \frac{1}{p^{s}} \rightarrow+\infty
$$

при $s \rightarrow 1+0$, т.е. бесконечность простых чисел $p$, принадлежащих арифметической прогрессии $n k+l, n=0,1,2 \ldots$. Замечу, что характеры Дирихле нашли многочисленные применения в самых разных областях математики.

Другим следствием развития идеи Эйлера явились работы 1848 г. Чебышева. Пользуясь соотношениями между $\zeta(s), \zeta^{\prime}(s)$, 
$\zeta^{\prime \prime}(s), \ldots$, Чебышев доказал теоремы о том, что если функция $\pi(x)$ - количество простых чисел, не превосходящих $x$, "хорошо" приближается функцией “типа" $\operatorname{Li}(x)$,

$$
\operatorname{Li}(x)=\int_{2}^{x} \frac{d t}{\ln t}
$$

то это будет $\operatorname{Li}(x)$, а также, что если существует предел отношения $\pi(x) / \operatorname{Li}(x)$ при $x \rightarrow+\infty$, то этот предел равен 1 . Первая теорема опровергала гипотезу Лежандра 1808 г. о том, что

$$
\pi(x) \sim \frac{x}{\ln x-1,08366 \ldots} .
$$

Другим выдающимся результатом Чебышева, который он получил под влиянием работ Эйлера, была теорема 1850 г.: при $x \geqslant x_{1}$ выполняются неравенства:

$$
0.9 \frac{x}{\ln x} \leqslant \pi(x) \leqslant 1.1 \frac{x}{\ln x} .
$$

Это второй, после теоремы Евклида, результат о поведении $\pi(x)$ при $x \rightarrow+\infty$, и первый результат о действительном порядке $\pi(x)$.

Тот факт, что $\pi(x)$, повидимому, лучше всего приближается функцией $\operatorname{Li}(x)$, в письме от 1849 г., которое было опубликовано много позднее (1863 г.), уже после работы Римана (1859 г.), сообщал Гаусс.

Наконец, Риман в 1859 г. рассмотрел $\zeta(s)$ как функцию комплексного переменного $s$, продолжил $\zeta(s)$ на всю $s$ - плоскость, доказал некоторые теоремы о $\zeta(s)$ как функции комплексного переменного, и установил связь между $\pi(x)$ и нулями $\zeta(s)$. Родилась наука "Теория дзета-функции Римана".

Скажу несколько слов об этой науке. Пусть $s-$ комплексное число, т.е. $s=\sigma+i t, i^{2}=-1, \sigma$ и $t$ - вещественные числа, $\sigma=\operatorname{Re} s$, $t=\operatorname{Im} s$.

При $\sigma=\operatorname{Re} s>1$ с помощью аналитических преобразований доказывается равенство:

$$
\pi^{-\frac{s}{2}} \Gamma\left(\frac{s}{2}\right) \zeta(s)=\frac{1}{s(s-1)}+\int_{1}^{\infty}\left(x^{\frac{s}{2}-1}+x^{-\frac{s+1}{2}}\right) \omega(x) d x,
$$

где $\Gamma(s)$ - гамма-функция Эйлера, $\omega(x)=\sum_{n=1}^{\infty} e^{-\pi x n^{2}}$. Из этого равенства видно, что функция $\xi(s)$,

$$
\xi(s)=s(s-1) \pi^{-\frac{s}{2}} \Gamma\left(\frac{s}{2}\right) \zeta(s),
$$


является целой функцией, т.е. голоморфной функцией в любом круге $|s|=R, R \rightarrow+\infty$. Таким образом, равенство (4) продолжает $\zeta(s)$ на всю $s$ - плоскость. Кроме того, из (4) следует равенство

$$
\xi(s)=\xi(1-s),
$$

которое называется "функциональным уравнением $\zeta(s)$ ”. Опять же из (4) нетрудно доказать, что $\xi(s)$ является целой функцией первого порядка и, следовательно, по теореме Вейерштрасса,

$$
\xi(s)=e^{a s} \prod_{\rho}\left(1-\frac{s}{\rho}\right) e^{\frac{s}{\rho}},
$$

где

$$
a=\ln 2+\ln \sqrt{\pi}-1-\frac{1}{2} \gamma=-0.023 \ldots,
$$

$\gamma$ - постоянная Эйлера, $\rho$ - бесконечная последовательность комплексных чисел вида $\rho=\beta+i \gamma_{1}, 0 \leqslant \beta \leqslant 1,0<\left|\gamma_{1}\right|$. Числа $\rho$ являются нулями $\zeta(s)$ ("нетривиальные нули").

В силу (6) числа $\rho$ расположены симметрично относительно прямых $\operatorname{Re} s=\frac{1}{2}, \operatorname{Im} s=0$, т.е. вместе с $\rho=\beta+i \gamma_{1}$ нулями $\zeta(s)$ будут числа $\bar{\rho}=\beta-i \gamma_{1}, \rho_{1}=1-\beta+i \gamma_{1}, \bar{\rho}_{1}=1-\beta-i \gamma_{1}$. Риман высказал гипотезу ("гипотеза Римана" или, коротко, $\mathrm{RH}$ ), что все $\rho$ лежат на прямой $\operatorname{Re} s=\frac{1}{2}$ ("критическая прямая"), т.е. $\operatorname{Re} \rho=\beta=\frac{1}{2}$.

Рассматривая логарифмическую производную функции $\xi(s)$ и пользуясь (5), (1), (7), при $\operatorname{Re} s>1$ получают равенство

$$
\sum_{n=2}^{\infty} \frac{\Lambda(n)}{n^{s}}=\frac{1}{s-1}-\sum_{\rho}\left(\frac{1}{s-\rho}+\frac{1}{\rho}\right)-\sum_{n=1}^{\infty}\left(\frac{1}{s+2 n}-\frac{1}{2 n}\right)-c_{1},
$$

где $\Lambda(n)$ - функция Мангольдта,

$$
\Lambda(n)=\left\{\begin{aligned}
\ln p, & \text { если } n=p^{k}, p-\text { простое число, } \\
0, & \text { если } n \neq p^{k},
\end{aligned}\right.
$$

$c_{1}$ - константа.

Из последней формулы методом "комплексного интегрирования" получается "явная формула" для функции Чебышева $\psi(x)$,

$$
\psi(x)=\sum_{n \leqslant x} \Lambda(n)=x-\sum_{\rho} \frac{x^{\rho}}{\rho}-\frac{\zeta^{\prime}(0)}{\zeta(0)}-\frac{1}{2} \ln \left(1-\frac{1}{x^{2}}\right)
$$


(если $x$ равняется степени простого числа, то последнее слагаемое в сумме $\psi(x)$ равняется $\left.\frac{1}{2} \Lambda(x)\right)$.

Функции $\pi(x)$ и $\psi(x)$ тесно связаны одна с другой, но $\psi(x)$ устроена проще, чем $\pi(x)$ : главный член ее асимптотической формулы есть $x$, в то время как главный член асимптотической формулы $\pi(x)$ есть $\operatorname{Li}(x)$. Соответственно, и формула для $\pi(x)$ через $\rho$ сложнее, чем (8) (“формула Римана”):

$$
\begin{gathered}
\pi(x)+\frac{1}{2} \pi(\sqrt{x})+\frac{1}{3} \pi(\sqrt[3]{x})+\cdots=\operatorname{Li}(x)-\sum_{\rho} \operatorname{Li}\left(x^{\rho}\right)-c, \\
\operatorname{Li}\left(x^{\rho}\right)=\operatorname{Li}\left(e^{\rho \ln x}\right), \quad \operatorname{Li}\left(e^{\omega}\right)=\int_{-\infty+i v}^{u+i v} \frac{1}{z} e^{z} d z, \quad \omega=u+i v,
\end{gathered}
$$

$c$ - константа.

Все основные применения $\zeta(s)$ к теории простых чисел связаны с (8) и ее вариантами.

Адамар и Валле-Пуссен в 1896 г. доказали, что $\operatorname{Re} \rho=\beta<1$ и, тем самым, что $\psi(x) \sim x, \pi(x) \sim \operatorname{Li} x$. Каждое из этих соотношений называется асимптотическим законом распределения простых чисел. Затем последовала целая серия результатов с уточнением границы нулей $\zeta(s)$, что сразу же вело к уточнению остаточного члена в асимптотических формулах $\psi(x)$ и $\pi(x)$ :

$$
\begin{aligned}
& \psi(x)=x+O(R(x)) ; \\
& \pi(x)=\operatorname{Li}(x)+O(R(x)), \quad R(x)=x \exp \left(-0.05(\ln x)^{\alpha}\right),
\end{aligned}
$$

$\alpha=\frac{1}{2}$ (Валле-Пуссен, 1898 г.); $\alpha=\frac{1}{2}\left(1+\frac{\ln \ln \ln x}{\ln \ln x}\right)$ (Литтлвуд, 1920 г.); $\alpha<\frac{4}{7}$ (Чудаков, 1936 г.); $\alpha<\frac{7}{12}$ (Коробов, 1957 г.); $\alpha<\frac{3}{5}$ (Виноградов, 1958 г.).

В этой же области исследований были доказаны исключительно интересные теоремы о простых числах, лежащих на коротких промежутках числовой оси (усиление постулата Бертрана):

$$
\pi(x+h)-\pi(x)=(1+o(1)) \frac{h}{\ln x}, \quad h=x^{\alpha},
$$

$\alpha=1-0.00003$ (Хохайзель, 1930 г.) $\alpha=1-0.004$ (Хейльбронн, 1933 г.) $\alpha=1-0.25$ (Чудаков, 1936 г.) $\alpha=1-\frac{3}{8}$ (Ингам, 1937 г.) $\alpha=1-\frac{2}{5}$ (Монтгомери, 1969 г.) $\alpha=1-\frac{5}{12}$ (Хаксли, 1972 г.).

Получено большое количество арифметических результатов при условии справедливости RH. Оказалось, что многие из этих 
результатов эквивалентны RH. В частности, соотношение вида

$$
R(x)=O\left(\sqrt{x} \ln ^{2} x\right)
$$

где $R(x)$ определяется равенством (9), эквивалентно RН. Получено много результатов о нулях $\zeta(s)$ : нули на критической прямой (Харди, Литтлвуд, Зигель, Кузьмин, Сельберг, Левинсон, Карацуба, Конри), "плотностные" теоремы (Ингам, Сельберг, Монтгомери, Хаксли), граница нулей (Валле-Пуссен, Литтлвуд, Чудаков, Коробов, Виноградов). Доказаны чисто функциональные теоремы о $\zeta(s)$ : теорема Гамбургера (1922 г.) о том, что $\zeta(s)$ однозначно определяется функциональным уравнением и тем, что она представляется рядом Дирихле при $\operatorname{Re} s>1$, теорема Воронина (1975 г.) об "универсальности" $\zeta(s)$ - в $\zeta(s)$ "содержатся" все аналитические функции. Исследованы классы функций, которые "похожи" на $\zeta(s)$ и для которых $\mathrm{RH}$ не имеет места; эти функции удовлетворяют функциональным уравнениям "риманова типа", т.е. вида (6), но не имеют "эйлерова" произведения вида (1) (Дэвенпорт, Хейльбронн, Сельберг, Воронин, Карацуба, Бомбьери, Хейчал). Для других функций, таких как $L(s, \chi), \zeta_{k}(s)$, которые, как и $\zeta(s)$, имеют Эйлерово произведение, повидимому, справедлив аналог RH (RHR - "расширенная" RH). Эти функции дали возможность решить ряд проблем о простых числах в арифметических прогрессиях (Линник, Родосский, БомбьериВиноградов), "почти бинарную" проблему Гольдбаха (Чен Жиран), "условно" решить проблему Гаусса-Артина о первообразных корнях (Хооли).

Невозможно перечислить в данном докладе даже малую часть тех результатов, которые получены к настоящему времени с помощью $\zeta(s)$ и ее обобщений.

9. Второй знаменитый источник содержится в главе XVI "O разбиении чисел на слагаемые”. Здесь в п. 302 (с. 263) Эйлер рассматривает произведение - дробь

$$
\frac{1}{\left(1-x^{\alpha} z\right)\left(1-x^{\beta} z\right)\left(1-x^{\gamma} z\right)\left(1-x^{\delta} z\right)\left(1-x^{\varepsilon} z\right) \ldots \text { и т.д. }}
$$

представляет его в виде

$$
1+P z+Q z^{2}+R z^{3}+S z^{4}+\cdots \text { и т.д. }
$$


и делает выводы о $P, Q, R, S, \ldots$. Мы поступим несколько иначе. Рассмотрим уравнение

$$
\alpha n+\beta m+\gamma l=N
$$

где $\alpha, \beta, \gamma$ - фиксированные натуральные числа, $N$ - целое неотрицательное число, $n, m, l$ - неизвестные неотрицательные числа. Пусть $I(N)$ - количество решений этого уравнения. Как найти $I(N)$ ? Рассматривая произведения $F(x)$,

$$
F(x)=\left(\sum_{n=0}^{\infty} x^{\alpha n}\right)\left(\sum_{m=0}^{\infty} x^{\beta m}\right)\left(\sum_{l=0}^{\infty} x^{\gamma l}\right),
$$

видим, что оно равно

$$
\sum_{N=0}^{\infty} I(N) x^{N}
$$

Если $|x|<1$, то каждая из сумм в (11), как сумма членов геометрической прогрессии, равна, соответственно,

$$
\frac{1}{1-x^{\alpha}}, \quad \frac{1}{1-x^{\beta}}, \quad \frac{1}{1-x^{\gamma}},
$$

т.е. получаем равенство (ср. с дробью Эйлера):

$$
F(x)=\sum_{N=0}^{\infty} I(N) x^{N}=\frac{1}{\left(1-x^{\alpha}\right)\left(1-x^{\beta}\right)\left(1-x^{\gamma}\right)} .
$$

Тем самым мы получили метод нахождения количеств решений уравнения (10), который сейчас называется методом "производящих" функций. Функция $F(x)$ - "производящая", коэффициенты ее ряда Тейлора при $x^{N}$ и есть $I(N)$ - нужные нам величины. Легко видеть, что

$$
I(N)=\left.\frac{1}{N !}\left(\frac{d^{N}}{d x^{N}} F(x)\right)\right|_{x=0} .
$$

При больших $N$ находить $I(N)$ по формуле (12) трудно. Эйлер, для нахождения $I(N)$ по этой формуле, представлял $F(x)$ в виде суммы простейших дробей. Но, после создания комплексного анализа, появилась другая формула для $I(N)$ - формула Коши:

$$
I(N)=\frac{1}{2 \pi i} \int_{|z|=R<1} \frac{F(z)}{z^{N+1}} d z .
$$


Эта формула нашла многочисленные применения в комбинаторном анализе. Одно из самых интересных ее применений - это теорема Харди-Рамануджана (1918 г.) о "разбиениях", т.е. о числе $p(n)$ решений уравнения

$$
n=x_{1}+2 x_{2}+3 x_{3}+4 x_{4}+\cdots,
$$

другими словами, о числе представлений $n$ в виде суммы всевозможных слагаемых. Производящей функцией здесь будет $f(x)$,

$$
f(x)=\prod_{k=1}^{\infty} \frac{1}{1-x^{k}}=\sum_{n=0}^{\infty} p(n) x^{n}, \quad|x|<1 .
$$

C помощью специальных преобразований, которые позднее послужили созданию "кругового метода", Харди и Рамануджан нашли асимптотику интеграла (13), отвечающего $f(x)$ и, тем самым, нашли асимптотическую формулу для $p(n)$ :

$$
\begin{gathered}
p(n)=\frac{e^{k \lambda_{n}}}{4 \sqrt{3} \lambda_{n}^{2}}\left(1+O\left(\frac{1}{\lambda_{n}}\right)\right), \\
k=\pi \sqrt{\frac{2}{3}}, \quad \lambda_{n}=\sqrt{n-\frac{1}{24}}, \quad n \geqslant 1 .
\end{gathered}
$$

Но это было только начало. Харди, Литтлвуд, Рамануджан (1920 г.) применили указанную идею к решению нелинейных аддитивных задач теории чисел, в частности, к проблеме Варинга, т.е. к проблеме изучения $I(N)$ - числа решений уравнения

$$
x_{1}^{n}+x_{2}^{n}+\cdots+x_{k}^{n}=N .
$$

в целых неотрицательных числах $x_{1}, x_{2}, \ldots, x_{k}$. Здесь производящей функцией $F(z),|z|<1$, будет такая:

$$
F(z)=\left(\sum_{x=0}^{\infty} z^{x^{n}}\right)^{k}
$$

совсем непростая функция. Но Харди-Литтлвуд-Рамануджан нашли метод, который позволял исследовать интеграл (13), отвечающий этой функции $F(z)$. Дугу окружности интегрирования в (13) они, по определенному правилу, разбивали на "большие" и "малые" дуги. На "больших" дугах получался "главный" член 
асимптотической формулы для $I(N)$. На "малых" дугах они оценили соответствующую часть $I(N)$ и доказали, что она есть омалое от "главного члена". Так было получено новое решение проблемы Варинга (проблему решил в 1909 г. Гильберт). Так появился "круговой" метод Харди-Литтлвуда-Рамануджана. Этот же метод был применен Харди-Литтлвудом (1923 г.) к тернарной проблеме Гольдбаха - к проблеме разрешимости в простых числах $p_{1}, p_{2}, p_{3}$ уравнения

$$
p_{1}+p_{2}+p_{3}=N .
$$

Принимая справедливой RHR, они доказали, что для количества $I(N)$ решений уравнения (15) справедлива асимптотическая формула:

$$
I(N) \sim \frac{N^{2}}{2(\ln N)^{3}} \sigma(N),
$$

где $\sigma(N)>1$, если $N$ - нечетное число. Отсюда следовало, что $I(N)>0$ при $N \geqslant N_{1}$, т.е. существование решений уравнения (15). Замечу, что в 1997 г. Дезуе, Эффингер, Риле и Зиновьев, пользуясь современными достижениями в аналитической теории чисел и мощными компьютерами, доказали, что в этой теореме $N_{1}=7$.

Начиная с 1929 г. к аддитивным проблемам теории чисел Виноградов стал применять тригонометрические суммы, т.е. суммы вида

$$
\sum_{x=1}^{N} e^{2 \pi i f(x)},
$$

где $f(x)$ - вещественнозначная функция. Основная идея применения тригонометрических сумм очень проста и опирается на формулу:

$$
\int_{0}^{1} e^{2 \pi i \alpha m} d \alpha= \begin{cases}1, & \text { если } m=0 ; \\ 0, & \text { если } m \neq 0, m \in \mathbb{Z} .\end{cases}
$$

Тогда вместо бесконечных рядов $\mathrm{F}(z)$ появляются конечные суммы. Например, $I(N)$ для (14) запишется так:

$$
\begin{gathered}
I(N)=\int_{0}^{1}(S(\alpha))^{k} e^{-2 \pi i \alpha N} d \alpha, \\
S(\alpha)=\sum_{x \leqslant \sqrt[n]{N}} e^{2 \pi i \alpha x^{n}} .
\end{gathered}
$$


Для уравнения (15) $I(N)$ запишется так:

$$
\begin{gathered}
I(N)=\int_{0}^{1} T^{3}(\alpha) e^{-2 \pi i \alpha N} d \alpha, \\
T(\alpha)=\sum_{p \leqslant N} e^{2 \pi i \alpha p} .
\end{gathered}
$$

Введение тригонометрических сумм не только упростило метод Харди-Литтлвуда-Рамануджана. Оно дало возможность решить ряд новых проблем теории чисел. Конечно, для этого надо было изучать суммы вида $S(\alpha), T(\alpha)$ и др. Это было сделано Виноградовым. Сначала он получил выдающиеся результаты в проблеме Варинга, в частности, доказав, что

$$
G(n)=O(n \ln n),
$$

где $G(n)$ - функция Харди-Литтлвуда - наименьшее $k$ такое, что каждое $N \geqslant N_{1}(n)$ представляется суммой $k$ слагаемых вида $x^{n}$, $x \in \mathbb{Z}, x \geqslant 0$. Очевидно, что $G(n)>n$, а верхняя оценка $G(n)$ у Харди-Литтлвуда-Рамануджана имела вид $G(n)<n 2^{n}$.

Затем Виноградов научился оценивать $|T(\alpha)|$ и в 1937 г. решил проблему Гольдбаха.

Тем самым проблема, поставленная в переписке Гольдбаха и Эйлера, была решена вследствие развития метода производящих функций Эйлера в круговой метод Харди-Литтлвуда-Рамануджана и метод тригонометрических сумм Виноградова.

Отмечу, что метод тригонометрических сумм является сейчас одним из основных методов аналитической теории чисел. Построена целая наука - теория тригонометрических сумм - с большим количеством результатов в ней, которые даже вкратце невозможно все перечислить здесь (аддитивные проблемы теории чисел, целые точки в областях на плоскости и в пространстве, распределение дробных долей вещественных функций, порядок роста арифметических рядов Дирихле, граница нулей $\zeta(s)$ и ее обобщений, осциллирующие интегралы в теории чисел, физике, теории вероятностей, математической статистике, томографии и многоемногое другое).

Заключение. К исследованиям Эйлера в области теории чисел тесно примыкают его работы по теории функций и математическому анализу. Достаточно назвать его гамма-функцию, интегралы первого и второго родов, выражение тригонометрических 
функций через экспоненциальные, формулу суммирования вида

$$
\begin{gathered}
\sum_{a<n \leqslant b} f(n)=\int_{a}^{b} f(x) d x+\rho(b) f(b)-\rho(a) f(a)-\int_{a}^{b} \rho(x) f^{\prime}(x) d x, \\
\rho(x)=\frac{1}{2}-\{x\},
\end{gathered}
$$

постоянную $\gamma$,

$$
\gamma=\lim _{n \rightarrow+\infty}\left(1+\frac{1}{2}+\frac{1}{3}+\frac{1}{4}+\cdots+\frac{1}{n}-\ln n\right),
$$

значения $\zeta(2 n), n=1,2,3, \ldots$,

$$
\zeta(2 n)=\alpha(n) \pi^{2 n}, \quad \alpha(n)-\text { рациональные числа, }
$$

в частности, формулу

$$
1+\frac{1}{4}+\frac{1}{9}+\frac{1}{16}+\frac{1}{25}+\cdots=\frac{\pi^{2}}{6},
$$

и многое другое.

Упомянутая параллель в творчестве Баха и Эйлера также, по моему мнению, не является случайным совпадением. Эйлер занимался математической теорией музыки и света. Ему принадлежат работы "Опыт новой теории музыки, ясно изложенной на основе вернейших начал гармонии", 1739 г., и "Теория образования голоса и музыки". В музыкальном энциклопедическом словаре, (см. [5], с. 650), об Эйлере сказано: "Занимался проблемами музыкальной акустики (объяснил происхождение обертонов) в связи с музыкальной теорией (аккордикой, модуляционностью), вопросами цветомузыкальных ассоциаций. Изданы в собр. соч., т. I, серия III ("Opera omnia", Lpz. - B., 1926)."

При написании статьи я пользовался материалом, содержащимся в следующих сочинениях:

\section{Список литературы}

[1] Леонард Эйлер, Введение в анализ бесконечно мальх, ОНТИ, М.Л., 1936.

[2] А. О. Гельфонд, “О некоторых характерных чертах идей Л. Эйлера в области математического анализа и его «Введении в анализ бесконечно малых»", УМН, 12:4 (1957), 29-39 Mi rm7662, MR 90533. 
[3] Развитие идей Леонарда Эйлера и современная наука, Сб. статей, ред. Н. Н. Боголюбов, Г. К. Михайлов, А. П. Юшкевич, Наука, М., 1988.

[4] Неопубликованные материаль Л. Эйлера по теории чисел, Отв. ред. Н. И. Невская, Наука, С.-Петербург, 1997.

[5] Музыкальный энииклопедический словарь, Советская энциклопедия, М., 1990.

Закончу статью словами Лапласа: “Читайте, читайте Эйлера, он учитель всех нас". 